$\mathrm{DTP} / 99 / 79$

November 1999

hep-th/9911058

\title{
Pasquier Models at $c=1$ : Cylinder Partition Functions and the role of the Affine Coxeter Element
}

\author{
Robert P. T. Talbot \\ Department of Mathematical Sciences, \\ University of Durham, Durham DH1 3LE, England円
}

\begin{abstract}
We calculate the partition functions of the affine Pasquier models on the cylinder in the continuum limit. We show that the partition function of any affine model may be expressed in terms of the orbit structure of the affine Coxeter element of the Weyl group associated with the defining graph of the model. Some of the consequences of this geometric relationship are explored.
\end{abstract}

${ }^{1}$ E-mail addresses: r.p.t.talbot@durham.ac.uk and/or r.p.talbot@gmx.net (permanent) 


\section{Introduction and Motivation}

The purpose of this paper is to find a general geometric expression for the partition functions of the affine Pasquier models on the cylinder. In so doing we find expressions for the partition functions of the models which are currently missing from the literature. In this section we present the problem and take the opportunity to introduce some notation.

The Pasquier models [19] are particular examples of graph-lattice models. They are defined on the square lattice with a height or spin function $\sigma$ assigning to each lattice vertex values within the set of nodes of a graph $\mathcal{G}$. The values of the heights of two neighbouring vertices are restricted so that they are adjacent nodes of $\mathcal{G}$. This restriction is encoded by the adjacency matrix of the graph which we shall write as $\mathcal{G}_{a b}$. The affine Pasquier models have graphs restricted to the set given in figure 1. These graphs (or to be more precise,

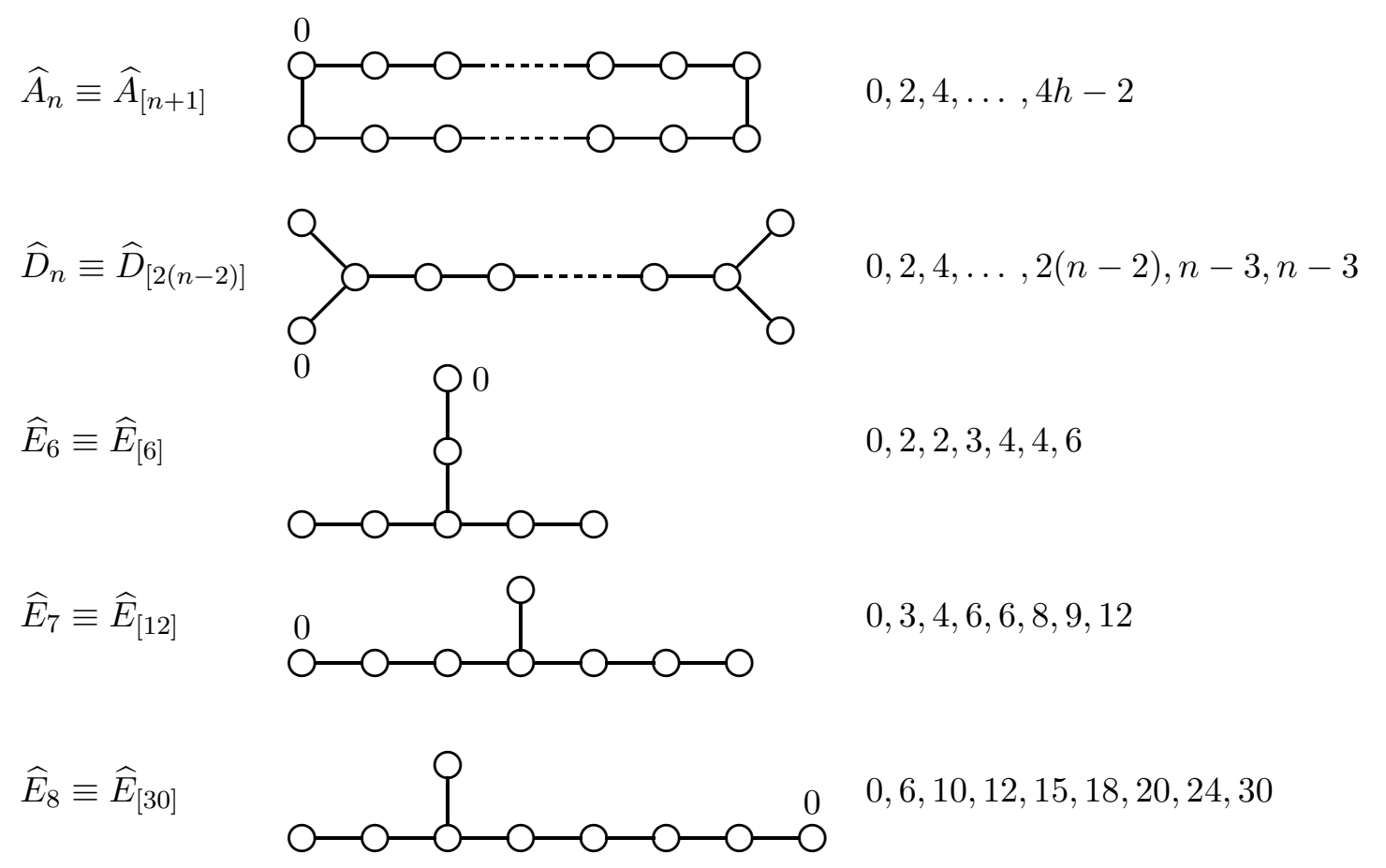

Figure 1: The affine graphs and their exponents. Each graph $\widehat{X}_{n}$ has $n+1$ nodes.

their adjacency matrices) are known to have highest eigenvalue $\beta \equiv \beta^{(0)}=2$. The models they provide are believed to be integrable and, at criticality, provide conformal theories with central charge $c=1$ (see references [20] and [11]). These graphs also arise in the classification of affine Kac-Moody algebras just as the classical (non-affine) $A D E$ Dynkin diagrams appear within the classification of Lie algebras. In fact each affine Kac-Moody algebra $\hat{\mathrm{g}}$ is related to an underlying Lie algebra $\overline{\mathrm{g}}$ known as its horizontal subalgebra. This relationship is reflected in the diagrams. Removing the "affine node" labelled 0 (see table (1) from the graph $\widehat{X}_{n}$ (say) associated with the affine algebra $\widehat{\mathrm{g}}$, one obtains the graph $X_{n}$ of the horizontal subalgebra $\bar{g}$.

We will examine models with a cylindrical rather than toroidal geometry. If opposing edges of a rectangular $M \times L$ lattice are identified so that the temporal direction is periodic then the partition function of the resultant model may be written in the continuum limit 
as 2

$$
\mathcal{Z}^{(\mathcal{G} ; a, b)}(q)=\sum_{n \in \mathcal{J}} \Lambda_{n}^{(\mathcal{G} ; a, b)} \chi_{\frac{n^{2}}{4}}(q)
$$

where the $\chi_{\underline{n^{2}}}(q)$ are $c=1$ Virasoro characters; and $\mathcal{J}$ is some index set labelling the representations of the single Virasoro algebra present in the theory. Note that to specify a model one must state the graph which defines its bulk behaviour and the boundary conditions on either end of the cylinder. Therefore we denote a model by $(\mathcal{G} ; a, b)$. In this paper we are only interested in the simple boundary conditions where the height function $\sigma$ is held to constant values $a, b \in \mathcal{G}$ on each end of the cylinder. We will denote by $\Gamma$ the discrete lattice on the cylinder.

The role played by the classical (i.e. non-affine) Coxeter element in the partition functions of the classical Pasquier models was first demonstrated by Dorey [6]. The Coxeter element is any one of a class of special elements within the Weyl group of reflections defined by the geometry of the simple roots. A Coxeter element is defined as the product of all the reflections associated with the simple roots (see [14 for a good overview). In practice we will choose a particular Coxeter element. If we exclude the graphs of $\widehat{A}$-type with odd numbers of nodes from our analysis, it follows from the $\mathbb{Z}_{2}$-colourability of the remaining affine graphs that the roots of an affine Kac-Moody algebra may be disjointly partitioned into two sets of mutually orthogonal roots $\Pi_{1}$ and $\Pi_{2}$. We fix notation by requiring $\alpha_{0}$ to be orthogonal to the roots of $\Pi_{2}$. Define the involutions $\omega_{1}$ and $\omega_{2}$ as the products of the reflections associated to the sets $\Pi_{1}$ and $\Pi_{2}$ of simple roots respectively. The exact ordering of the roots within either of these sets is irrelevant as they are mutually orthogonal. Then the Steinberg-ordered Coxeter element [24] is defined as

$$
\omega \stackrel{\text { def }}{=} \omega_{2} \omega_{1} .
$$

It is this particular choice of Coxeter element that we will find easiest to manipulate.

The generalisation to the affine Coxeter element is non-trivial however. The order of (any of) the Coxeter elements is known as the Coxeter number. In the affine cases, this order is infinite and an analogous number known as the affine Coxeter number $h$ (finite) takes its place in expressions for eigenvalues and eigenvectors of the adjacency matrix. (We will often find it convenient to label graphs and their eigenvectors and eigenvalues with this number in which case we will do so by placing square parentheses around the Coxeter number, $\mathcal{G}_{[h]}$ for example.) The affine Weyl group $\widehat{W}$ contains translations as well as ordinary reflections. We shall see that the action of the Affine Coxeter element can be thought of as the action of something not unlike the action of the Coxeter element in the root space of the underlying horizontal algebra (this action has finite order $h$ ) combined with the additional effect produced by a translation. There are also "imaginary" roots, pointwise invariant under $\widehat{W}$ complicating the geometry. A good overview of the relationship between affine Weyl groups and Kac-Moody algebras may be found in [10].

Dorey [6] demonstrated that the coefficients $\Lambda_{n}^{(\mathcal{G} ; a, b)}$ in the expression analogous to (1.1) for the non-affine models $(c<1)$ had a simple geometric interpretation in terms of the orbit structure of the (non-affine analogue of the) Coxeter element (1.2). We extend this result to the affine models $(c=1)$ and in so doing also provide an alternative method of proof of the result for the non-affine cases. We will also be interested in the geometric consequences of the relationship mentioned earlier between an affine Kac-Moody algebra and its underlying Lie algebra. 
We begin in the next section by calculating the coefficients $\Lambda_{n}^{\left(\widehat{A}_{[2 h]} ; a, b\right)}$ for the model $\left(\widehat{A}_{[2 h]} ; a, b\right)$. In section 3 we construct affine generalisations of intertwiners which will relate the remaining $\widehat{A}-, \widehat{D}$ - and $\widehat{E}$-based models to this basic model. We show that all the partition functions have a single algebraic form in section 4 . In section 5 , we rewrite this form in terms of the geometry of the Weyl group of reflections and finally, in section 6 we explore some of the consequences of this geometric interpretation.

\section{The Partition Function of the Model $\left(\widehat{A}_{n} ; a, b\right)$}

We consider first the model $\left(A_{\infty} ; a, b\right)$. The height function, $\sigma$, of this model is the discrete version of a continuous free field variable $\varphi \in \mathbb{R}$. Up to rescaling of $\varphi$, this theory has partition function

$$
\mathcal{Z}^{\left(A_{\infty} ; a, b\right)}(M, L)=\int_{\substack{\varphi\left(a^{0}, 0\right)=a \\ \varphi\left(a^{0}, L\right)=b \\ \varphi\left(a^{0}+M, a^{1}\right)=\varphi\left(a^{0}, a^{1}\right)}} \mathrm{D} \varphi \exp \left\{-\frac{\pi g}{4} \int \mathrm{d}^{2} a\left|\nabla \varphi\left(a^{0}, a^{1}\right)\right|^{2}\right\},
$$

where $g=1$ [21]. This model is well known in the literature to provide a $c=1$ conformal field theory (see [12] for example). The equivalence,

$$
\lim _{\substack{d \rightarrow 0 \\ M, L \rightarrow \infty \\ M / L \text { fixed }}} \mathcal{Z}_{\Gamma}^{\left(A_{\infty} ; a, b\right)}=\mathcal{Z}^{\left(A_{\infty} ; a, b\right)}(M, L),
$$

of the lattice model in the continuum limit to the Gaussian or Coulomb-gas model (2.1) is established by examining the renormalisation-group flows of the so-called (unrestricted) solid-on-solid model mapped onto the $A_{\infty}$ model via a set of transformations [18].

The partition function (2.1) is calculated by splitting $\varphi\left(a^{0}, a^{1}\right)=\varphi_{0}\left(a^{0}, a^{1}\right)+\varphi\left(a^{1}\right)$. The field $\varphi_{1}\left(a^{1}\right)$ is the classical solution to the equations of motion. The field $\varphi_{0}$ is periodic in the time direction and satisfies the boundary conditions $\varphi_{0}\left(a^{0}, 0\right)=\varphi_{0}\left(a^{0}, L\right)=0$. By zeta-regularisation [21],

$$
\begin{aligned}
& \int_{\substack{\varphi\left(a^{0}, 0\right)=\varphi_{0}\left(a^{0}, L\right)=0 \\
0}} \mathrm{D} \varphi_{0} \exp \left\{-\frac{\pi g}{4} \int \mathrm{d}^{2} a\left|\nabla \varphi_{0}\right|^{2}\right\}=\eta^{-1}(q), \\
& \varphi_{0}\left(a^{0}+M, a^{1}\right)=\varphi_{0}\left(a^{0}, a^{1}\right)
\end{aligned}
$$

with $q \equiv \exp (-M \pi / L)$, the modular parameter.

The classical field $\varphi_{1}\left(a^{1}\right)=(b-a) a^{1} / L$ [21], so (2.1) is

$$
\mathcal{Z}^{\left(A_{\infty} ; a, b\right)}(q)=\eta^{-1}(q) \exp \left\{-\frac{\pi g}{4}\left(\frac{b-a}{L}\right)^{2} M L\right\}=\eta^{-1}(q) q^{g(b-a)^{2} / 4} .
$$

The model is (manifestly) invariant under shifts in the boundary conditions $(a, b) \rightarrow$ $(a+c, b+c)$, so we set $\varepsilon \equiv b-a$; thus, setting also $g=1$,

$$
\mathcal{Z}^{\left(A_{\infty} ; 0, \varepsilon\right)}(q)=\eta^{-1}(q) q^{\varepsilon^{2} / 4}
$$


By graph-symmetry, the partition function of the model $\left(\widehat{A}_{2 h-1} ; a, b\right)$ is also invariant under 'translation' of the boundary conditions. We again rewrite the boundary condition as $\varepsilon \equiv b-a$. The continuum limit of the partition function is found by noting that the height function $\sigma$ is here the discrete version of the continuous field $\varphi \in \mathbb{R} / 2 h \mathbb{Z}$; i.e. the field $\varphi$ of (2.1) is now compactified on a circle of circumference $2 h$ [9]. With a suitable rescaling of the field $\varphi$, the continuum partition function will be given by (2.1), again with $g=1$. The partition function (2.1) is evaluated by again splitting $\varphi\left(a^{0}, a^{1}\right)=$ $\varphi_{0}\left(a^{0}, a^{1}\right)+\varphi_{1}\left(a^{1}\right)$. The field $\varphi_{0}\left(a^{0}, a^{1}\right)$ is as before and provides the contribution (2.3). The classical field $\varphi_{1}\left(a^{1}\right)$ is modified to identify the boundary condition $\varepsilon$ with $\varepsilon+2 n h$ for $n \in \mathbb{Z}$. Thus

$$
\varphi_{1}\left(a^{1}\right) \equiv \sum_{n=-\infty}^{\infty} \varphi_{1}^{(n)}\left(a^{1}\right)
$$

where $\varphi_{1}^{(n)}\left(a^{1}\right)$ is the classical field in the $n^{\text {th }}$ instanton sector and is

$$
\varphi_{1}^{(n)}\left(a^{1}\right)=(\varepsilon+2 n h) \frac{a^{1}}{L} .
$$

Thus the continuum partition function of the model $\left(\widehat{A}_{2 h-1} ; 0, \varepsilon\right)$ is given by the result (2.5) with the identification $\varepsilon \equiv \varepsilon+2 h$, i.e.

$$
\mathcal{Z}^{\left(\widehat{A}_{2 h-1} ; 0, \varepsilon\right)}(q)=\eta^{-1}(q) \sum_{n \in \mathbb{Z}} q^{\frac{(\varepsilon+2 n h)^{2}}{4}} .
$$

The partition functions $(2.5)$ and $(2.8)$ can be placed in the form (1.1) by noting that

$$
\eta^{-1}(q) q^{\frac{n^{2}}{4}}=\sum_{p=0}^{\infty} \chi_{\frac{(n+2 p)^{2}}{4}}(q)
$$

where $n$ is a positive integer and $\chi_{\underline{n^{2}}}(q)$ are degenerate $c=1$ characters (refer to references [7] and [15] for details). If we define the generalised semi-infinite Kronecker comb as

$$
\Omega_{n, \varepsilon}^{\left(a_{1}, \ldots, a_{k}\right)} \equiv \sum_{m_{1}, \ldots, m_{k}=0}^{\infty} \delta_{n, \varepsilon+\sum_{i=1}^{k} m_{i} a_{i}},
$$

where $\delta$ is the usual Kronecker- $\delta$; then (2.5) is of the form (1.1) with

$$
\Lambda_{n}^{\left(A_{\infty} ; 0, \varepsilon\right)}=\Omega_{n, \varepsilon}^{(2)} .
$$

Similarly, defining $\varepsilon \equiv \varepsilon+2 n h$, it is an easy exercise to see that (2.8) is of the form (1.1) with

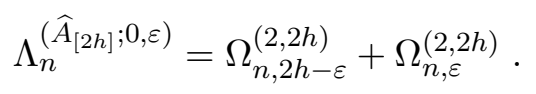

We could deduce the partition functions of the models based upon the odd cycles, $\widehat{A}_{[2 h-1]}$, by substituting $2 h \mapsto 2 h-1$. However the $\widehat{A}_{[2 h]}$-based models form a kind of "basis set" as we shall see below. 


\section{Intertwiners Between Affine Models}

We now have an expression (2.12) for the partition function of the models of the form $\left(\widehat{A}_{[2 h]} ; 0, \varepsilon\right)$. We construct the partition functions of $\widehat{D}$ - and $\widehat{E}$-based models and of the odd cycle $\widehat{A}$ models by re-expressing these models, still on the finite lattice $\Gamma$, in terms of

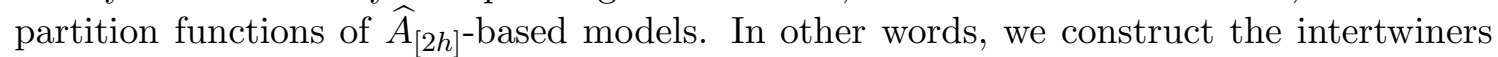
interrelating the models. These relationships between the models continue to hold in the continuum limit and we then may use (2.12) to construct explicit partition functions for the $\widehat{A}_{[2 h-1]^{-}}, \widehat{D}$ - and $\widehat{E}$-based cases.

We wish to find coefficients $N_{0 \lambda ; h^{\prime}}^{a b}$ such that

$$
\mathcal{Z}_{\Gamma}^{\left(\mathcal{G}_{[h]} ; a, b\right)}=\sum_{h^{\prime}} \sum_{\lambda \in \widehat{A}_{\left[h^{\prime}\right]}} N_{0 \lambda ; h^{\prime}}^{a b} \mathcal{Z}_{\Gamma}^{\left(\widehat{A}_{\left[h^{\prime}\right]} ; 0, \lambda\right)}
$$

Denote by $\operatorname{Tr}_{(a, b)}$ the trace subject to the boundary conditions $(a, b)$. The Markov trace is defined for any operator $\mathrm{Y}$ on the Hilbert space of a model based on the graph $\mathcal{G}$ by

$$
\begin{aligned}
\operatorname{Tr}_{\sigma_{1}}^{(\nu)} \mathrm{Y} & \stackrel{\text { def }}{=} \sum_{\sigma_{L}} \frac{\psi_{\sigma_{L}}^{(\nu)}}{\psi_{\sigma_{1}}^{(\nu)}} \underset{\left(\sigma_{1}, \sigma_{L}\right)}{\operatorname{Tr}} \mathbf{Y} \\
& \equiv \sum_{\sigma_{2}, \ldots, \sigma_{L}} \frac{\psi_{\sigma_{L}}^{(\nu)}}{\psi_{\sigma_{1}}^{(\nu)}}\left\langle\sigma_{1}, \ldots, \sigma_{L}|\mathrm{Y}| \sigma_{1}, \ldots, \sigma_{L}\right\rangle ;
\end{aligned}
$$

where the $\left\{{\underset{\sim}{\psi}}^{(\mu)} \mid \mu \in \mathrm{v}^{*}(\mathcal{G})\right\}$ are the eigenvectors of the graph $\mathcal{G}$ labelled by the exponents of $\mathcal{G}$. The modified partition function is defined [23] in terms of this trace by

$$
\begin{aligned}
\mathcal{Z}_{\Gamma}^{[\mathcal{G} ; \nu]} & \stackrel{\text { def }}{=} \operatorname{Tr}_{a}^{(\nu)} T^{M} \\
& \equiv \sum_{b \in \mathcal{G}} \frac{\psi_{b}^{(\nu)}}{\psi_{a}^{(\nu)}} \mathcal{Z}_{\Gamma}^{(\mathcal{G} ; a, b)} .
\end{aligned}
$$

Sochen [23] has demonstrated the remarkable property that this partition function is both independent of $a$ and that it is graph independent: any two graphs, $\mathcal{G}_{1}$ and $\mathcal{G}_{2}$, sharing the same exponent $\nu$ and highest (Perron-Frobenius) eigenvector $\beta$ have identical modified partition functions on the lattice $\Gamma$, i.e.

$$
\mathcal{Z}_{\Gamma}^{\left[\mathcal{G}_{1} ; \nu\right]}=\mathcal{Z}_{\Gamma}^{\left[\mathcal{G}_{2} ; \nu\right]}
$$

The solution to this equation (3.4), with $\mathcal{G}_{1}$ and $\mathcal{G}_{2}$ both chosen to be (non-affine) $A D E$ graphs with equal Coxeter number yields the intertwiners between the $A$-, $D$ - and $E$-based models. Although typically no $\widehat{A}$ graph shares all the exponents of any given $\widehat{D}$ or $\widehat{E}$ graph, the graph independence of (3.3) can still be used to interrelate the affine models as follows: Let $\left\{\beta^{(\mu)} \mid \mu \in \mathrm{v}^{*}(\mathcal{G})\right\}$ denote the eigenvalues of $\mathcal{G}$ and let

$$
\mathcal{Z}_{\Gamma}^{\left[\mathcal{G}_{[h]} ; \nu\right]}=f\left(\beta, \beta^{(\nu)}\right)
$$

where $f$ is some graph independent function, denote the modified partition function for a model based on the affine graph $\mathcal{G}$ with affine Coxeter number $h$ (see [23]). We seek 
solutions to the more general equation

$$
\mathcal{Z}_{\Gamma}^{\left[\mathcal{G}_{[h]} ; \nu\right]}=\sum_{\substack{h^{\prime} \in \mathbb{Z}^{+} \\ \nu^{\prime} \in \widehat{A}_{\left[h^{\prime}\right]}}} X_{h^{\prime} \nu^{\prime}}^{h \nu} \mathcal{Z}_{\Gamma}^{\left[\widehat{A}_{\left[h^{\prime}\right]} ; \nu^{\prime}\right]}
$$

We allow freedom both in the choice of the $\widehat{A}$ graph (labelled by its Coxeter number $h^{\prime}$ ) and in the exponent $\nu^{\prime}$. Substituting in (3.5) and labelling the eigenvalues of a graph with Coxeter number $k$ by $\beta_{[k]}^{(\mu)}$, we therefore seek a solution for the $X_{h^{\prime} \nu^{\prime}}^{h \nu}$ such that,

$$
f\left(\beta_{[h]}, \beta_{[h]}^{(\nu)}\right)=\sum_{\substack{h^{\prime} \in \mathbb{Z}^{+} \\ \nu^{\prime} \in \widehat{A}_{\left[h^{\prime}\right]}}} X_{h^{\prime} \nu^{\prime}}^{h \nu} f\left(\beta_{\left[h^{\prime}\right]}, \beta_{\left[h^{\prime}\right]}^{\left(\nu^{\prime}\right)}\right) .
$$

By property (3.4), the unknown function $f$ is identical on each side for any given fixed lattice $\Gamma$.

Given the eigenvalues $\beta_{[h]}$ and $\beta_{[h]}^{\nu}$ on the left hand side of (3.7) we can solve the equation by choosing the eigenvalues $\left\{\beta_{\left[h^{\prime}\right]}^{\left(\nu^{\prime}\right)}\right\}$ on the right hand side appropriately. Examining the general form of the eigenvalues $\beta_{[k]}^{(\mu)}=2 \cos \frac{\mu \pi}{k}$ one might naïvely attempt to choose $h^{\prime}=h$ and $\nu^{\prime}=\nu$. However, this would require that the exponent set of the graph $\mathcal{G}_{[h]}$ be a subset of the exponent set of the graph $\widehat{A}_{[h]}\left(\right.$ i.e. $\left.\mathrm{v}^{*}\left(\mathcal{G}_{[h]}\right) \subseteq \mathrm{v}^{*}\left(\widehat{A}_{[h]}\right)\right)$ and, as mentioned already, this is not the case in general. (For example, $\widehat{D}_{5}$ has the odd exponent 3 occurring with multiplicity 2 . However, not only are there no $\widehat{A}$-based models with odd exponents, but no exponent of any $\widehat{A}$ graph has a multiplicity exceeding 1.) Fortunately we may circumnavigate this inconvenience by choosing instead $h^{\prime}=2 h$ and $\nu^{\prime}=2 \nu$, i.e.

$$
X_{h^{\prime} \nu^{\prime}}^{h \nu}=\delta_{h^{\prime}}^{2 h} \delta_{\nu^{\prime}}^{2 \nu}
$$

This provides a solution to (3.6). Substituting (3.8) and (3.3) into (3.6) we obtain,

$$
\sum_{b \in \mathcal{G}_{[h]}} \frac{\psi_{b}^{(\nu)}}{\psi_{a}^{(\nu)}} \mathcal{Z}_{\Gamma}^{\left(\mathcal{G}_{[h]} ; a, b\right)}=\sum_{d \in \widehat{A}_{[2 h]}} \frac{\phi_{d}^{(2 \nu)}}{\phi_{c}^{(2 \nu)}} \mathcal{Z}_{\Gamma}^{\left(\widehat{A}_{[2 h]} ; c, d\right)}
$$

where the $\left\{{\underset{\sim}{(\mu)}}^{(\mu)}\right\}$ are eigenvectors of $\mathcal{G}_{[h]}$ and the $\left\{\underset{\sim}{\phi^{(\mu)}}\right\}$ eigenvectors of $\widehat{A}_{[2 h]}$. Both $a$ and $c$ may be chosen arbitrarily on the respective graphs. Assuming the $\left\{{\underset{\sim}{(\mu)}}^{(\mu)}\right\}$ have been chosen to be an orthonormal set, this is inverted to yield,

$$
\mathcal{Z}_{\Gamma}^{\left(\mathcal{G}_{[h]} ; a, b\right)}=\sum_{\lambda \in \widehat{A}_{[2 h]}}\left\{\sum_{\mu \in \mathrm{v}^{*}(\mathcal{G})} \frac{\phi_{\lambda}^{(2 \mu)}}{\phi_{0}^{(2 \mu)}} \psi_{a}^{(\mu) *} \psi_{b}^{(\mu)}\right\} \mathcal{Z}_{\Gamma}^{\left(\widehat{A}_{[2 h]} ; 0, \lambda\right)} ;
$$

where we set $c=0$ (the affine node) without loss of generality. This is of the form (3.1) with

$$
N_{0 \lambda ; h^{\prime}}^{a b}=\delta_{h^{\prime}, 2 h} N_{\lambda}^{a b}
$$


and

$$
N_{\lambda}^{a b} \equiv \sum_{\mu \in \mathrm{v}^{*}(\mathcal{G})} \frac{\phi_{\lambda}^{(2 \mu)}}{\phi_{0}^{(2 \mu)}} \psi_{a}^{(\mu) *} \psi_{b}^{(\mu)}
$$

We remark that although complete freedom was permitted in the $\widehat{A}$ models appearing in equation (3.1), (3.10) demonstrates that the even cycles provide a basis set from which all the other models may be constructed.

Equation (3.11), together with equation (3.12) clearly provide a solution to the decomposition (3.1) but not necessarily the only one. In general there are a number of different possible solutions. However, any non-trivial solution is suitable for our purposes and we do not investigate other possibilities here.

The coefficients (3.12) are the analogues of the classical intertwiners

$$
V_{a b}^{\lambda} \equiv \sum_{\mu \in \mathrm{v}^{*}(\mathcal{G})} \frac{\phi_{\lambda}^{(\mu)}}{\phi_{1}^{(\mu)}} \psi_{a}^{(\mu) *} \psi_{b}^{(\mu)} .
$$

They possess a similar algebraic form and play a similar role. They differ immediately chiefly in that they relate a model with affine Coxeter number $h$ to a model with affine Coxeter number $2 h$ and even interrelate $\widehat{A}$ models! In contrast, intertwiners interrelate classical models with the Coxeter number preserved in the relation.

It is certainly tempting, given the form $\phi_{[h] \varepsilon}^{(\mu)}=\cos \frac{\mu \pi \varepsilon}{h}$ for the eigenvectors of the $\widehat{A}_{[h]}$ graph, to identify the coefficients (3.12) with the classical intertwiners (3.13). Denote by square-parentheses the Coxeter number of the graph to which a vector refers. Then, naïvely

$$
\frac{\phi_{[2 h] \lambda}^{(2 \mu)}}{\phi_{[2 h] 0}^{(2 \mu)}} \equiv \frac{\phi_{[h] \lambda}^{(\mu)}}{\phi_{[h] 0}^{(\mu)}} .
$$

However the terms on the right-hand side of this equality are not defined unless $\mu$ is even (i.e. an exponent of the $\widehat{A}$ graph); the more general summation over the full exponent set of the graph $\mathcal{G}$ implies that this is not always the case. Furthermore, upon examination, the coefficients (3.12) appear to be both half-integral and of either sign; properties not shared by (3.13).

We remark that for the $\widehat{D}$ and $\widehat{E}$ cases, the coefficient $N_{\lambda}^{a b}$ of (3.12) will be zero if the $\mathbb{Z}_{2}$-parity of the length of the path between $a$ and $b$ on $\mathcal{G}_{[h]}$ is not the same as the

parity of the path between 0 and $\lambda$ on $\widehat{A}_{[2 h]}$. The argument is quite complicated and we do not repeat it here (it is detailed in full in [26]). Thus if the original model $\mathcal{G}_{[h]}$ has even (odd) boundary conditions (i.e. the path length between $a$ and $b$ is even (odd)) then the $\widehat{A}$-models onto which it decomposes will also have strictly even (odd) boundary conditions.

\section{A Simple Form for the Partition Functions}

While it is possible at this stage to calculate the intertwiners $(3.12)$ and thereby the partition functions, we shall instead show that the partition functions may be unified into a single simple form. For the explicit calculation of the intertwiners and from these the partition functions, the interested reader is referred elsewhere [26]. 
It has been noted (see for example [8] or [9]) that the intertwiners (3.13) interrelating the classical (i.e. non-affine) Pasquier models may be written as Chebychev polynomials of the second kind. These polynomials $\left\{\mathcal{U}^{(n)}(x) \mid n \in \mathbb{Z}\right\}$ are defined by the recursion relation

$$
\mathcal{U}^{(n)}(x)=x \mathcal{U}^{(n-1)}(x)-\mathcal{U}^{(n-2)}(x) ;
$$

together with two 'initial conditions': the values of two subsequent polynomials in the series. We are concerned only with the series of polynomials generated by the initial conditions:

$$
\mathcal{U}^{(0)}(x)=1, \quad \mathcal{U}^{(1)}(x)=x ;
$$

and we note that this implies that $\mathcal{U}^{(-1)}(x)=0$. The classical intertwiners $(3.13)$ may be written

$$
V_{a b}^{\lambda} \equiv \mathcal{U}_{a b}^{(\lambda-1)}(\mathcal{G})
$$

We propose the same form for the affine intertwiners (3.12). We prove that this is indeed the case now:

Lemma 1 The coefficient $\Lambda_{n}^{\varepsilon} \equiv \Lambda_{n}^{\left(\widehat{A}_{[2 h]} ; 0, \varepsilon\right)}$ of $\chi_{\frac{n^{2}}{4}}(q)$ in the expansion (1.1) of the partition function of the model $\left(\widehat{A}_{[2 h]} ; 0, \varepsilon\right)$ is given by

$$
\Lambda_{n}^{\varepsilon}=\mathcal{U}_{0 \varepsilon}^{(n)}\left(\widehat{A}_{[2 h]}\right)
$$

In other words, it is given by the $n^{\text {th }}$-Chebychev polynomial of the second kind satisfying $\mathcal{U}^{(0)}(\widehat{A})=\mathbb{1}$ and $\mathcal{U}^{(1)}(\widehat{A})=\widehat{A}$.

Proof The proof is by induction on the variable $n$.

We first establish that the initial conditions (4.2) for the Chebychev recursion relation are satisfied. We observe, directly from (2.12), that

$$
\Lambda_{0}^{\varepsilon}=\delta_{0}^{\varepsilon}=\mathcal{U}_{0 \varepsilon}^{(0)}\left(\widehat{A}_{[2 h]}\right)
$$

and

$$
\Lambda_{1}^{\varepsilon}=\delta_{1}^{\varepsilon}+\delta_{2 h-1}^{\varepsilon}=\mathcal{U}_{0 \varepsilon}^{(1)}\left(\widehat{A}_{[2 h]}\right)
$$

as required.

Having established that the initial conditions are satisfied, we now examine the recursion relation (4.1). Assume that $\exists N \in \mathbb{Z}^{+}$such that $\forall 2 \leq n<N$ that (4.4) holds. Then

$$
\begin{aligned}
{\left[\mathcal{U}^{(N-1)}\left(\widehat{A}_{[2 h]}\right) \widehat{A}_{[2 h]}-\mathcal{U}^{(N-2)}\left(\widehat{A}_{[2 h]}\right)\right]_{0 \varepsilon} } & =\sum_{\lambda \in \widehat{A}_{[2 h]}} \Lambda_{N-1}^{\lambda} \widehat{A}_{\lambda \varepsilon}-\Lambda_{N-2}^{\varepsilon} \\
& =\Lambda_{N-1}^{\varepsilon-1}+\Lambda_{N-1}^{\varepsilon+1}-\Lambda_{N-2}^{\varepsilon}
\end{aligned}
$$

using the induction hypothesis. Note that, as defined in section 2 , the $\Lambda_{n}^{\varepsilon}$ have a period $2 h$ in the variable $\varepsilon$, so that addition and subtraction in this variable (such as in (4.7)) are understood to be taken modulo $2 h$. 


\begin{tabular}{|l||cccccc|cccccc|ccc|}
\hline \multicolumn{1}{|c||}{$\varepsilon$} & \multicolumn{11}{c|}{$n$} \\
\hline & $\mathbf{0}$ & 1 & 2 & 3 & 4 & 5 & $\mathbf{6}$ & 7 & 8 & 9 & 10 & 11 & $\mathbf{1 2}$ & 13 & 14 \\
\hline \hline & & & & & & & & & & & & & & & \\
4 & $\mathbf{0}$ & & 1 & & $\mathbf{2}$ & & $\mathbf{2}$ & & 3 & & $\mathbf{4}$ & & $\mathbf{4}$ & & 5 \\
5 & & 1 & & 1 & & $\mathbf{2}$ & & 3 & & 3 & & $\mathbf{4}$ & & 5 & \\
\hline 0 & 1 & & 1 & & 1 & & 3 & & 3 & & 3 & & 5 & & 5 \\
1 & & 1 & & 1 & & $\mathbf{2}$ & & 3 & & 3 & & $\mathbf{4}$ & & 5 & \\
2 & $\mathbf{0}$ & & 1 & & $\mathbf{2}$ & & $\mathbf{2}$ & & 3 & & $\mathbf{4}$ & & $\mathbf{4}$ & & 5 \\
3 & & $\mathbf{0}$ & & $\mathbf{2}$ & & $\mathbf{2}$ & & $\mathbf{2}$ & & $\mathbf{4}$ & & $\mathbf{4}$ & & $\mathbf{4}$ & \\
4 & $\mathbf{0}$ & & 1 & & $\mathbf{2}$ & & $\mathbf{2}$ & & 3 & & $\mathbf{4}$ & & $\mathbf{4}$ & & 5 \\
5 & & 1 & & 1 & & $\mathbf{2}$ & & 3 & & 3 & & $\mathbf{4}$ & & 5 & \\
\hline 0 & 1 & & 1 & & 1 & & 3 & & 3 & & 3 & & 5 & & 5 \\
1 & & 1 & & 1 & & $\mathbf{2}$ & & 3 & & 3 & & $\mathbf{4}$ & & 5 & \\
& & & & & & & & & & & & & & & \\
\hline
\end{tabular}

Figure 2: The coefficients $\Lambda_{n, \varepsilon}$ for the model $\widehat{A}_{5}$. The multiples of $n=h \equiv 6$ are highlighted in bold. Due to 'parity constraints', every second value is automatically zero and these values have been left blank for clarity. The values in the figure are 'coloured' alternatively bold and normal to emphasise the "embedded-diamond"-like pattern of the numbers. Note that the figure is periodic in the variable $\varepsilon$ (see text) and that $n$ takes values from 0 to $\infty$. This same basic pattern of numbers occurs for each of the $\mathbb{Z}_{2}$-colourable $\widehat{A}$-based models.

The righthand side of (4.7) will be equal to the required $\Lambda_{N}^{\varepsilon}$ iff

$$
\Lambda_{N-1}^{\varepsilon-1}+\Lambda_{N-1}^{\varepsilon+1}=\Lambda_{N}^{\varepsilon}+\Lambda_{N-2}^{\varepsilon} .
$$

This equation is identically true $\forall N$ replaced with $n>2$ as a detailed analysis of the coefficients $\Lambda_{n}^{\varepsilon}$ shows. If we examine a typical grid of values $\Lambda_{n}^{\varepsilon}$ (given by equation (2.12)), as in figure 2. Fixing arbitrarily, the values of $\varepsilon$ and $n$ we see that $\Lambda_{n}^{\varepsilon}$ takes the value, $m$ say, with coordinates $\varepsilon$ and $n$ in the grid, $\Lambda_{n-2}^{\varepsilon}$ takes the values two spaces to the left (or 'west') of this value, $\Lambda_{n-1}^{\varepsilon-1}$ takes the value 'northwest' and $\Lambda_{n-1}^{\varepsilon+1}$ the value 'southwest'. Due to the periodicity of the variable $\varepsilon$ and the "embedded-diamond"-like pattern the numbers $\Lambda_{n}^{\varepsilon}$ follow (for any value of even $h$ ) one of the following is always the case:

$$
\begin{aligned}
& \Lambda_{n}^{\varepsilon}=\Lambda_{n-2}^{\varepsilon}=\Lambda_{n-1}^{\varepsilon-1}=\Lambda_{n-1}^{\varepsilon+1}=m ; \\
& \Lambda_{n}^{\varepsilon}=\Lambda_{n-1}^{\varepsilon-1}=m, \quad \Lambda_{n-1}^{\varepsilon+1}=\Lambda_{n-2}^{\varepsilon}=m-1 ; \\
& \Lambda_{n}^{\varepsilon}=\Lambda_{n-1}^{\varepsilon+1}=m, \quad \Lambda_{n-1}^{\varepsilon-1}=\Lambda_{n-2}^{\varepsilon}=m-1 ; \\
& \Lambda_{n}^{\varepsilon}=m, \quad \Lambda_{n-2}^{\varepsilon}=m-2, \quad \Lambda_{n-1}^{\varepsilon-1}=\Lambda_{n-1}^{\varepsilon+1}=m-1 ;
\end{aligned}
$$

with $m$ some positive integer and in each such case, the identity (4.8) holds.

This concludes the proof. 
Theorem 2 The coefficients of the Virasoro characters in the expansion (1.1) for all the affine models are given by the Chebychev polynomials of equation (4.12). In other words

$$
\sum_{\substack{\mu \in \mathrm{v}^{*}(\mathcal{G}) \\ \varepsilon \in \widehat{A}_{[2 h]}}} \frac{\phi_{\varepsilon}^{(2 \mu)}}{\phi_{0}^{(2 \mu)}} \psi_{a}^{(\mu) *} \psi_{b}^{(\mu)} \Lambda_{n}^{\varepsilon}=\mathcal{U}_{a b}^{(n)}(\mathcal{G}) .
$$

Here $\mathcal{U}^{(n)}(\mathcal{G})$ denotes the Chebychev polynomial of the second kind satisfying: $\mathcal{U}^{(0)}(\mathcal{G})=\mathbb{1}$ and $\mathcal{U}^{(1)}(\mathcal{G})=\mathcal{G}$ and $\Lambda_{n}^{\varepsilon}$ again denotes the coefficient $\Lambda_{n}^{\left(\widehat{A}_{[2 h]} ; 0, \varepsilon\right)}$.

Proof This follows by induction on $n$ using lemma 1, the eigenvalue property

$$
\beta_{[2 h]}^{(2 \mu)}=\beta_{[h]}^{(\mu)},
$$

and the identity

$$
\sum_{\lambda} \widehat{A}_{[h] \varepsilon \lambda} F(\lambda)=F(\varepsilon-1(\bmod h))+F(\varepsilon+1(\bmod h)) .
$$

Thus we arrive at the following general form for the partition functions of each of the affine Pasquier models on the cylinder:

$$
\mathcal{Z}^{(\mathcal{G} ; a, b)}(q)=\sum_{n=0}^{\infty} \chi_{\frac{n^{2}}{4}}(q) \mathcal{U}_{a b}^{(n)}(\mathcal{G}) .
$$

\section{The Role of Coxeter Geometry}

We now establish the role played in the partition functions by the Steinberg-ordered affine Coxeter element defined in section 1 (equation (1.2)). Given the definition of this Coxeter element, we exclude the models based on the odd cycles $\widehat{A}_{[2 h-1]}$ from our analysis.

The Coxeter elements associated with any classical algebra are known to be mutually conjugate and therefore possess identical spectra [14]. The question of conjugacy of the affine Coxeter elements was addressed by Berman et al. [1] and also by Coleman [3]. In the case of the affine tree graphs, i.e. the $\widehat{D}$ - and $\widehat{E}$-cases, it can be proved that all Coxeter elements are conjugate (see also [14]). Since there is no special choice of Coxeter element, we choose the most convenient example to manipulate. However, in the case of the cycles, the $\widehat{A}$-graphs, the Coxeter elements do fall into a number of distinct spectral classes. Indeed, the Coxeter elements associated with the graph $\widehat{A}_{n-1}$ fall into $[n / 2]$ spectral classes. In particular they do not belong all to a single conjugacy class. Regardless, we will find the affine generalisation of the Steinberg-ordered Coxeter element to be the most useful even in this case. It possesses the property of being a representative of the largest spectral class. When $n$ is even, this largest spectral class is unique and might be taken to define a natural choice of sorts although we do not make use of this property. In any case, we remark that any Coxeter element and its inverse, both belong to the same spectral class.

Let $\widehat{\mathrm{g}}$ denote an affine Kac-Moody algebra with Coxeter-Dynkin diagram $\mathcal{G}$ (with $l+1$ nodes). Let $\bar{g}$ denote its horizontal subalgebra. We denote by $\Pi \equiv\left\{\alpha_{a} \mid a \in 0,1, \ldots l\right\}$ 
the set of simple roots with $\alpha_{0}$ the affine root and by $\left\{\rho_{a} \mid a \in 0,1, \ldots l\right\}$ the basis of fundamental weights. We denote by $\sigma_{a}$ the Weyl-reflection defined by $\alpha_{a}$ so that

$$
\lambda \mapsto \sigma_{\alpha} \lambda \stackrel{\text { def }}{=} \lambda-\left\langle\lambda, \alpha^{\vee}\right\rangle \alpha,
$$

where $\langle$,$\rangle denotes the inner (scalar) product within \widehat{\mathrm{h}}^{*}$ and $\alpha^{\vee} \equiv 2 \alpha /\langle\alpha, \alpha\rangle$ the coroot of $\alpha$. The behaviour of the weights under Weyl reflections is given by

$$
\sigma_{a} \rho_{b}=\rho_{b}-\delta_{a b} \alpha_{b}
$$

as the weights and the coroots are dual. We recall (see [10 for example) that the roots of an affine Kac-Moody algebra may be written in the form $(\bar{\alpha}, k, d)$ where $\bar{\alpha}$ is a root of $\overline{\mathrm{g}}$ and $k$ and $d$ are respectively the components w.r.t. the centre and derivation of $\widehat{\mathrm{g}}$. Denote the roots of $\widehat{g}$ by $\widehat{\Phi}$. A subset of $\widehat{\Phi}$ may be identified with the root system $\bar{\Phi}$ of $\bar{g}$ by

$$
(\bar{\alpha}, 0,0) \equiv \bar{\alpha} .
$$

The positive roots $\widehat{\Phi}^{+}$of $\widehat{g}$ are

$$
\widehat{\Phi}^{+} \stackrel{\text { def }}{=}\left\{\alpha=(\bar{\alpha}, 0, n) \in \widehat{\Phi} \mid n>0 \text { or } n=0, \bar{\alpha} \in \bar{\Phi}^{+}\right\},
$$

and the negative roots $\widehat{\Phi}^{-} \equiv \widehat{\Phi} \backslash \widehat{\Phi}^{+}$. We use the notation $\alpha>0$ for positive roots and $\alpha<0$ for negative roots. Given the choice (5.4), simple roots exist and are

$$
\alpha_{i}=\left(\bar{\alpha}_{i}, 0,0\right)=\bar{\alpha}_{i} \quad \text { for } i=1, \ldots, l \text {; }
$$

where $\bar{\Pi} \equiv\left\{\bar{\alpha}_{a} \mid a \in \mathcal{G} \backslash\{0\}\right\}$ are the simple roots of $\bar{\Phi}$; together with

$$
\alpha_{0}=(-\bar{\theta}, 0,1)=\delta-\bar{\theta} .
$$

$\bar{\theta}$ is the highest root of $\overline{\mathrm{g}}$ and $\delta \equiv(0,0,1)$. Note that $\delta$ is an imaginary or lightlike root and is invariant under the action of $\widehat{W}$.

If we regard $\mathcal{G}$ as an operator on the root space $\widehat{\mathrm{h}}^{*}$ of $\widehat{\mathrm{g}}$, i.e.

$$
\begin{aligned}
\mathcal{G}: \widehat{\mathrm{h}}^{*} & \rightarrow \widehat{\mathrm{h}}^{*}, \\
\alpha_{a} & \mapsto \sum_{b \in \mathcal{G}} \mathcal{G}_{a b} \alpha_{b} .
\end{aligned}
$$

then it may be shown that

Lemma 3 (Kostant [17]) The action of $\mathcal{G}$ regarded as an operator on $\widehat{\mathrm{h}}^{*}$ is

$$
\mathcal{G}=\omega_{1}+\omega_{2}
$$

where $\omega_{1}$ and $\omega_{2}$ are the involutions defined in section 1 .

This (5.8) may be substituted into (4.12) to obtain a geometric expression for the partition functions. Performing the calculation however, it is not immediately obvious what sort of relationship we actually have. Instead we proceed in a different way: 
Choose the following as orbit representatives for the Steinberg-ordered Coxeter element $\omega$ defined in section 샤

$$
\phi_{a} \stackrel{\text { def }}{=}\left(1-\omega^{-1}\right) \rho_{a}
$$

or equivalently, by (5.2) and (1.2),

$$
\phi_{a}= \begin{cases}\alpha_{a} & ; \text { if } \alpha_{a} \in \Pi_{1} . \\ \omega_{1} \alpha_{a} & ; \text { if } \alpha_{a} \in \Pi_{2} .\end{cases}
$$

This choice is a generalisation of the representatives chosen by Kostant 16 for the orbit representatives of the non-affine (Steinberg-ordered) Coxeter element. As it is a generalisation it is easy to see that this set is also linearly independent. Furthermore, their orbits are distinct; indeed if one supposes [5] that $\phi_{b}$ lies in the Coxeter orbit of another: i.e. $\omega^{p} \phi_{a}=\phi_{b}$ for some values of $a, b$ and $p$; then, by (5.9),

$$
\omega^{p} \rho_{a}=\rho_{b} .
$$

This cannot be true as all fundamental weights are dominant highest weights and are therefore not related to each other by any Weyl element 13. The representatives (5.9) are uniquely characterised as the positive roots which go negative (i.e. whose images are negative roots) under the action of the (affine) Coxeter element $\omega$. Now

Proposition 4 The action of the operator $\mathcal{G}$ (see equation (5.7)) on the orbit representatives $\widehat{R} \equiv\left\{\phi_{a} \mid a \in 0,1, \ldots, l\right\}$, is given by

$$
\sum_{c \in \mathcal{G}} \phi_{c} \mathcal{G}_{c b}=\left\{\begin{array}{ll}
\left(1+\omega^{-1}\right) \phi_{b} & ; \text { if } \alpha_{b} \in \Pi_{1} . \\
(1+\omega) \phi_{b} & ; \text { if } \alpha_{b} \in \Pi_{2} .
\end{array} .\right.
$$

Proof The proof is by simple calculation using definition (5.9), equation (5.2) and lemma 3 .

In analogy with [6], we introduce the function

$$
v_{a} \equiv \begin{cases}0 & ; \text { if } \alpha_{a} \in \Pi_{2} . \\ 1 & ; \text { if } \alpha_{a} \in \Pi_{1} .\end{cases}
$$

and define $v_{a b} \equiv v_{a}-v_{b}$.

We now demonstrate the connection between the Chebychev polynomials and the affine Coxeter element. Dorey [6] demonstrated using explicit forms for the eigenvectors in the intertwiners (3.13) that the partition functions of the classical Pasquier models could be expressed as

$$
\mathcal{Z}^{(\mathcal{G} ; a, b)}(q)=\sum_{\bar{\omega}^{-p} \bar{\phi}_{b} \in \bar{\Phi}^{+}} \chi_{1,2 p+1+v_{a b}}^{c}(q)\left(\bar{\rho}_{a}, \bar{\omega}^{-p} \bar{\phi}_{b}^{\vee}\right)
$$

The quantities $\bar{\rho}_{a}, \bar{\omega}$ and $\bar{\phi}_{b}$ refer to the root system and Weyl group of a classical Lie algebra $\overline{\mathrm{g}}$ with adjacency matrix $\mathcal{G}$ and Coxeter number $\bar{h}$; the scalar product $($,$) is the$ 
usual Euclidean bilinear form and the Virasoro characters $\chi_{a, b}^{c}(q)$ refer to representations of the conformal algebra with central charge $c=1-\frac{6}{\bar{h}(\bar{h}-1)}<1$. As remarked upon earlier (demonstrated in the affine case), intertwiners and the Chebychev polynomials are related. Thus we are led to conjecture a similar form for the affine partition functions by equating the natural affine generalisation of the inner product appearing in (5.14) to the Chebychev polynomials of the affine adjacency matrices.

Theorem 5 Let $v_{a b}$ be defined as above (5.13). If $\mathcal{G}$ is the Coxeter-Dynkin diagram of the Kac-Moody algebra $\widehat{\mathrm{g}}$ and $\langle$,$\rangle the affine bilinear form (scalar product) on the maximal$ Cartan subalgebra $\widehat{h}$ of $\widehat{g}$; then

$$
\mathcal{U}_{a b}^{\left(2 p+v_{a b}\right)}(\mathcal{G})=\left\langle\rho_{a}, \omega^{-p} \phi_{b}^{\vee}\right\rangle ;
$$

$\omega$ denotes the (Steinberg-ordered affine) Coxeter element defined in equation (1.2).

Proof The proof is by induction on the variable $N$ in $\mathcal{U}^{(N)}(\mathcal{G})$. The proof splits into four subproofs corresponding to the four different ways of assigning the roots $\alpha_{a}$ and $\alpha_{b}$ amongst the sets $\Pi_{1}$ and $\Pi_{2}$. In each case we first check that the form $\left\langle\rho_{a}, \omega^{-p} \phi_{b}^{\vee}\right\rangle$ reproduces the correct initial conditions (4.1), i.e. $\mathcal{U}^{(0)}(\mathcal{G})=\mathbb{1}$ and $\mathcal{U}^{(1)}(\mathcal{G})=\mathcal{G}$. This is done using the duality between the weights and the (co-)roots and the fact that the affine scalar product $\langle$,$\rangle is normal, i.e.$

$$
\left\langle\eta \lambda, \eta \lambda^{\prime}\right\rangle=\left\langle\lambda, \lambda^{\prime}\right\rangle \quad \forall \eta \in \widehat{W} ; \lambda, \lambda^{\prime} \in \widehat{\mathrm{h}}^{*} .
$$

The induction hypothesis in each case may be shown using repeated use of proposition 4 . The full details of the proof may be found in [26].

Thus we rewrite equation (4.12) as

$$
\mathcal{Z}^{(\mathcal{G} ; a, b)}=\sum_{p=p^{\prime}}^{\infty} \chi_{\frac{\left(2 p+v_{a b}\right)^{2}}{4}}(q)\left\langle\rho_{a}, \omega^{-p} \phi_{b}^{\vee}\right\rangle
$$

with:

$$
p^{\prime}=\left\{\begin{array}{ll}
0 & ; \text { if } v_{a b}=0 \text { or } 1 \\
1 & ; \text { if } v_{a b}=-1
\end{array} .\right.
$$

This expresses the affine partition functions in terms of the affine Coxeter element. Note that the summation automatically excludes those terms which are zero due to the requirement that $|a-b|$ and $n$, now replaced by $2 p+v_{a b}$, be both even or both odd.

We remark that [6] demonstrated the form (5.14) for the classical models by an entirely different method. We duplicate this result using the method just described by recalling that the intertwiner $V_{a b}^{\lambda}$ relating the $\mathcal{G}=D, E$ models to an $A$ model is equivalent to a Chebychev polynomial by equation (4.3). Theorem 5 holds in the classical case also once one replaces the affine scalar product $\langle$,$\rangle with the usual one ($,$) and the affine Coxeter$ transformation, weights and orbit representatives with their classical analogues. Thus we may equate the intertwiner with a geometric expression. The result (5.14) follows directly from [22]

$$
\mathcal{Z}^{(\mathcal{G} ; a, b)}(q)=\sum_{\lambda=1}^{h-1} \chi_{1, \lambda}^{c}(q) V_{a b}^{\lambda}
$$




\section{Geometrical Consequences}

In analogy with [6], we may attempt to construct invariant subspaces under the action of $\omega$ as follows:

Define

$$
\alpha_{i}^{(\mu)} \stackrel{\text { def }}{=} \sum_{\substack{a \\ \text { such that } \\ \alpha_{a} \in \Pi_{i}}} \psi_{a}^{(\mu)} \alpha_{a} \quad \text { for } i=1,2 .
$$

These vectors would appear to possess the properties:

$$
\begin{aligned}
& \omega_{i} \alpha_{i}^{(\mu)}=-\alpha_{i}^{(\mu)}, \\
& \omega_{j} \alpha_{i}^{(\mu)}=\alpha_{i}^{(\mu)}+\beta^{(\mu)} \alpha_{j}^{(\mu)} \quad \text { for } i \neq j .
\end{aligned}
$$

So that $V^{(\mu)} \stackrel{\text { def }}{=} \operatorname{span}\left\{\alpha_{1}^{(\mu)}, \alpha_{2}^{(\mu)}\right\}$ is closed.

Unfortunately, for some values of $\mu$ and $i$, such vectors (6.1) may be identically zero; this can be verified by explicit calculation. However, for $\mu=0$ it is easy to see that the properties of the Perron-Frobenius eigenvector ensure that $\alpha_{1}^{(0)}$ and $\alpha_{2}^{(0)}$ are both non-zero, distinct and linearly independent. Hence the $\mu=0$ invariant subspace exists. Choose the Perron-Frobenius eigenvector to have all coefficients positive. We change notation and write

$$
\alpha^{(i)}=\sum_{\substack{a \\ \text { such that } \\ \alpha_{a} \in \Pi_{i}}} \psi_{a}^{(0)} \alpha_{a}
$$

So that

$$
\omega_{i} \alpha^{(j)}=\left\{\begin{array}{ll}
-\alpha^{(j)} & \text {;if } i=j . \\
\alpha^{(j)}+2 \alpha^{(i)} & ; \text { if } i \neq j .
\end{array} .\right.
$$

We also define the weights

$$
\rho^{(i)}=\sum_{\substack{a \\ \text { such that } \\ \alpha_{a} \in \Pi_{i}}} \psi_{a}^{(0)} \rho_{a} .
$$

Their action upon the $\left\{\alpha^{(i)}\right\}$ is

$$
\left\langle\rho^{(i)}, \alpha^{(j) \vee}\right\rangle=\frac{1}{2} \delta^{i j}
$$

and upon roots in general, their action is to project onto the spaces $\operatorname{span}\left\{\alpha^{(1)}\right\}$ and $\operatorname{span}\left\{\alpha^{(2)}\right\}$ respectively. Equation $\left(\sqrt{6.6)}\right.$ indicates that $2 \rho^{(i)}$ is the dual of $\alpha^{(i) \vee}$; therefore the projection operator $\mathcal{J}$ onto the space $V \equiv \operatorname{span}\left\{\alpha^{(1)}, \alpha^{(2)}\right\}$ is given by

$$
\mathcal{J} \lambda \equiv 2\left\langle\rho^{(1)}, \lambda^{\vee}\right\rangle \alpha^{(1)}+2\left\langle\rho^{(2)}, \lambda^{\vee}\right\rangle \alpha^{(2)}
$$

where $\lambda \equiv(\bar{\lambda}, 0, n)$ is any root. In particular $\mathcal{J}^{2}=\mathcal{J}$. We let $\alpha \equiv \alpha^{(1)}+\alpha^{(2)}=\psi_{0}^{(0)} \delta$ and $\rho \equiv \rho^{(1)}+\rho^{(2)}$ so that $\langle\rho, \alpha\rangle=1$. 
We remark that $\rho$ as defined, is a positive integer sum over the weights $\left\{\rho_{a} \mid a \in \mathcal{G}\right\}$. Using the fact that any root may be written as a positive or negative integer sum of the simple roots; we see that, by analogy with [6],

$$
\begin{aligned}
& \lambda \text { is a positive root } \\
& \Leftrightarrow\left\langle\rho_{a}, \lambda^{\vee}\right\rangle>0 \text { for any } a \\
& \Leftrightarrow\left\langle\rho, \lambda^{\vee}\right\rangle>0 .
\end{aligned}
$$

Thus we have:

Lemma 6 Any root of $\widehat{\Phi}$ is a positive root, iff the scalar product of its coroot with $\rho$ is positive; i.e.

$$
\lambda \equiv(\bar{\lambda}, 0, n) \in \widehat{\Phi}^{+} \quad \text { iff } \quad\left\langle\rho, \lambda^{\vee}\right\rangle>0
$$

The following result is perhaps obvious; however we state it for clarity:

Proposition 7 The projection $\mathcal{J}$ commutes with the Weyl group operators $\omega_{1}$ and $\omega_{2}$; and hence with the Coxeter element. That is

$$
\left.\left[\mathcal{J}, \omega_{i}\right]\right|_{\widehat{\Phi}}=0, \quad \text { for } i=1,2
$$

insofar as they act upon the roots $\widehat{\Phi}$.

Proof The simple roots $\widehat{\Pi}$ provide a basis for $\widehat{\Phi}$. Therefore one need only examine to see if $\mathcal{J} \omega_{i} \alpha_{a}=\omega_{i} \mathcal{J} \alpha_{a}$ for each $a$ and $i$. This follows easily using the properties (6.4) and the definition (6.7).

For convenience, for any operator $\mathrm{Y}$, let

$$
\llbracket Y \rrbracket_{b} \stackrel{\text { def }}{=}\left\{\begin{array}{ll}
\mathbb{1} & \text {; if } \alpha_{b} \in \Pi_{1} . \\
Y & ; \text { if } \alpha_{b} \in \Pi_{2} .
\end{array} .\right.
$$

We also define

$$
P(a) \stackrel{\text { def }}{=}\left\{\begin{array}{ll}
1 & ; \text { if } \alpha_{a} \in \Pi_{1} . \\
2 & ; \text { if } \alpha_{a} \in \Pi_{2} .
\end{array} .\right.
$$

Theorem 8 For all $a, b \in \mathcal{G}$ and $p \in \mathbb{Z}^{+}$:

$$
\left\langle\rho_{a}, \omega^{-p} \phi_{b}^{\vee}\right\rangle \geq 0
$$


Proof By lemma 6, we need only establish that $\left\langle\rho, \omega^{-p} \phi_{b}\right\rangle>0$.

By proposition 7 :

$$
\begin{aligned}
\mathcal{J} \omega^{-p} \phi_{b} & =\omega^{-p} \llbracket \omega_{1} \rrbracket_{b} \mathcal{J} \alpha_{b} \\
& =2\left\langle\rho^{(1)}, \alpha_{b}^{\vee}\right\rangle \omega^{-p} \llbracket \omega_{1} \rrbracket_{b} \alpha^{(1)}+2\left\langle\rho^{(2)}, \alpha_{b}^{\vee}\right\rangle \omega^{-p} \llbracket \omega_{1} \rrbracket_{b} \alpha^{(2)} \\
& =2\left\langle\rho^{(P(b))}, \alpha_{b}^{\vee}\right\rangle \omega^{-p} \llbracket \omega_{1} \rrbracket_{b} \alpha^{(P(b))} ;
\end{aligned}
$$

where we make use of the fact that $\left\langle\rho^{(i)}, \alpha_{b}^{\vee}\right\rangle=0$ unless $\alpha_{b} \in \Pi_{i}$. Thus we need only examine the Coxeter orbits of $\alpha^{(1)}$ and $\omega_{1} \alpha^{(2)} \equiv 2 \alpha^{(1)}+\alpha^{(2)}$.

We see from (6.4) that the application of $\omega^{-1}$ to $(n) \alpha^{(1)}+(n-1) \alpha^{(2)}$ yields $(n+$ 2) $\alpha^{(1)}+(n+1) \alpha^{(2)}$; i.e. $n$ is replaced by $n+2$. The roots of both orbits follow this form. Hence, as $\mathcal{J}$ projects onto the invariant space $V$,

$$
\begin{aligned}
\left\langle\rho, \omega^{-p} \phi_{b}^{\vee}\right\rangle & =\left\langle\rho, \mathcal{J} \omega^{-p} \phi_{b}^{\vee}\right\rangle \\
& = \begin{cases}2\left\langle\rho^{(1)}, \alpha_{b}^{\vee}\right\rangle(2 p+1) & ; \text { when } \alpha_{b} \in \Pi_{1} . \\
2\left\langle\rho^{(2)}, \alpha_{b}^{\vee}\right\rangle(2 p+3) & ; \text { when } \alpha_{b} \in \Pi_{2} .\end{cases}
\end{aligned}
$$

This may be rewritten as

$$
\left\langle\rho, \omega^{-p} \phi_{b}^{\vee}\right\rangle=2\left\langle\rho, \alpha_{b}^{\vee}\right\rangle\left(2 p+1+2 \delta_{2, P(b)}\right) .
$$

The scalar product appearing on the RHS clearly exceeds zero as $\alpha_{b}$ is a simple root and hence a positive root. The remaining factor is positive for all $p \geq 0$.

This completes the proof.

Thus the coefficients $\Lambda_{n}^{(\mathcal{G} ; a, b)}$ appearing in the expression (1.1) are all positive. That they are integers follows trivially from the duality of the weights and coroots together with the fact that the set $\widehat{\Phi}$ is closed under the action of $\widehat{W}$.

We mention as an aside that it might be interesting to investigate the relationship, if any, between the projection $\mathcal{J}$ and the defect map investigated by Berman et al. [1].

Much of the structure of an affine algebra $\widehat{g}$ is related to the underlying structure of the horizontal subalgebra $\bar{g}$. If we separate out the translation part of the action from the affine Coxeter element we should be able to see some of the structure of this subalgebra. Indeed, consider the translation $t$ defined by

$$
\mathrm{t} \stackrel{\text { def }}{=} \sigma_{0} \sigma_{\bar{\theta}}
$$

where $\bar{\theta}$ is the highest root of $\overline{\mathrm{g}}$. The action of $\mathrm{t}$ on any $\lambda \equiv(\bar{\lambda}, k, d)$ is

$$
\mathrm{t} \lambda=\left(\bar{\lambda}-k \bar{\theta}^{\vee}, k, d-\left(\bar{\lambda}, \bar{\theta}^{\vee}\right)-\frac{2}{(\bar{\theta}, \bar{\theta})} k\right) ;
$$

and in particular, for any root $\alpha \equiv(\bar{\alpha}, 0, n)$,

$$
\begin{aligned}
\mathrm{t} \alpha & =\left(\bar{\alpha}, 0, n-\left(\bar{\alpha}, \bar{\theta}^{\vee}\right)\right) \\
& =\alpha-\left(\bar{\alpha}, \bar{\theta}^{\vee}\right) \delta ;
\end{aligned}
$$

i.e. it 'translates' any real root by $-\left(\bar{\alpha}, \bar{\theta}^{\vee}\right)$ in the lightlike direction. As $\sigma_{0}=\mathrm{t} \sigma_{\bar{\theta}}$ we may regard $\widehat{W}$ as being generated by the set

$$
\{\mathrm{t}\} \cup\left\{\sigma_{a} \mid a=1, \ldots, l\right\} .
$$


$\widehat{W}$ is the smallest group containing both the (classical) Weyl group $\bar{W}$ of $\bar{g}$ and the affine translation t. Define $\check{\omega}_{2}$ by the requirement

$$
\omega_{2}=\mathrm{t} \check{\omega}_{2}=\check{\omega}_{2} \mathrm{t}^{-1}
$$

so that $\check{\omega}_{2}$ is $\omega_{2}$ with the reflection $\sigma_{0}$ replaced with $\sigma_{\bar{\theta}}$. Define

$$
\begin{gathered}
\check{\omega}_{1} \stackrel{\text { def }}{=} \omega_{1} \\
\text { and } \quad \check{\omega} \stackrel{\text { def }}{=} \check{\omega}_{2} \check{\omega}_{1} ;
\end{gathered}
$$

so that $\omega \equiv \mathrm{t} \check{\omega}$. Note in particular that $\check{\omega}, \check{\omega}_{1}$ and $\check{\omega}_{2}$ are all elements of the Weyl group $\bar{W}$ of the horizontal subalgebra $\bar{g}$ and are therefore constrained to be of finite order and to act trivially on the lightlike components of the roots of $\widehat{g}$.

We may use this "Euclidean Coxeter element" $\breve{\omega}$ to examine separately the action of $\omega$ on the horizontal and lightlike parts of the orbit representatives. Consider

$$
\begin{aligned}
\omega^{-1} \phi_{b} & =\omega^{-1}\left(\bar{\phi}_{b}, 0, \delta_{0 b}\right) \\
& =\check{\omega}^{-1} \mathrm{t}\left(\bar{\phi}_{b}, 0, \delta_{0 b}\right) \\
& =\check{\omega}^{-1}\left(\bar{\phi}_{b}, 0, \delta_{0 b}+\left(\bar{\phi}_{b}, \bar{\theta}^{\vee}\right)\right) \\
& =\left(\check{\omega}^{-1} \bar{\phi}_{b}, 0, \delta_{0 b}+\left(\bar{\phi}_{b}, \bar{\theta}^{\vee}\right)\right) .
\end{aligned}
$$

Iterating, so that for $p \geq 0$,

$$
\omega^{-p} \phi_{b}=\left(\check{\omega}^{-p} \bar{\phi}_{b}, 0, \delta_{0 b}+\sum_{p^{\prime}=0}^{p-1}\left(\check{\omega}^{-p^{\prime}} \bar{\phi}_{b}, \bar{\theta}^{\vee}\right)\right) .
$$

As $\check{\omega}$ has period $h$ (this is the definition of the affine Coxeter number, see [25] and [1]), we may write

$$
\omega^{-(q h+r)} \phi_{b}=\left(\check{\omega}^{-r} \bar{\phi}_{b}, 0, \delta_{0 b}+\sum_{r^{\prime}=0}^{r-1}\left(\check{\omega}^{-r^{\prime}} \bar{\phi}_{b}, \bar{\theta}^{\vee}\right)+q \sum_{r^{\prime}=0}^{h-1}\left(\check{\omega}^{-r^{\prime}} \bar{\phi}_{b}, \bar{\theta}^{\vee}\right)\right) ;
$$

where $p=q h+r$ and $r<h$ by the usual division algorithm. Thus to calculate the coefficient $\left\langle\rho_{a}, \omega^{-p} \phi_{b}\right\rangle$ we need only examine the behaviour of the "Euclidean projection" of the Coxeter orbits onto $\bar{\Phi}$, the root system of $\bar{g}$. In particular, due to the periodicity of $\breve{\omega}$, we find that one can calculate any coefficient from the knowledge of the $h(l+1)$ vectors

$$
\left\{\check{\omega}^{-r} \bar{\phi}_{b} \mid r \in \mathbb{Z}, 0 \leq r \leq h-1 ; b \in \mathcal{G}\right\}
$$

together with their inner products with the (dual of the) highest root $\bar{\theta}^{\vee}$; in particular, the sums

$$
\left\{\Delta_{b}^{(r)} \stackrel{\text { def }}{=} \sum_{r^{\prime}=0}^{r-1}\left(\check{\omega}^{-r^{\prime}} \bar{\phi}_{b}, \bar{\theta}^{\vee}\right) \mid r \in \mathbb{Z}, 0 \leq r \leq h-1\right\}
$$

determine the action of the translation $\mathrm{t}$ which is localised to the lightlike components of $\omega^{-p} \phi_{b}$. The action of $\omega$ is thus periodic on the horizontal components of the roots 
but translates the lightlike components. Note that it is the orbits $\langle\breve{\omega}\rangle \bar{\phi}_{b}$ of the Euclidean Coxeter element which appear in these quantities and not the orbits of the Coxeter element $\bar{\omega}$ of the algebra $\bar{g}$. However it is interesting to see that the entire structure of the affine Coxeter orbits can be determined by examining the orbits of $\check{\omega}$ alone. These being of finite cardinality, allow one to determine the entire partition function (5.17) from a finite number of calculations. Indeed this method of calculation was checked using Mathematica. The correct partition functions are obtained.

\section{Concluding Remarks}

We have shown that the partition functions of the $\left(\mathbb{Z}_{2}\right.$-colourable $)$ affine Pasquier models may be expressed in terms of the orbit structure of the affine Coxeter element (see equation (5.17)). We have thus generalised the geometric expression of [6] from $c<1$ conformal models to $c=1$.

In particular we have found expressions, (4.12) and (5.17) (the former valid for all the models), for the partition functions of the affine Pasquier models with fixed boundary conditions on the cylinder which until now had been missing from the literature. The interested reader may like to verify that, in the specific case of the 4-state Potts model with free boundary conditions, the partition function implied by (4.12) or (5.17) agrees with that found in [22].

The geometric expression (5.17), whilst unlike (4.12) is not manifestly symmetric in the boundary conditions, permits an easy verification of the positive integrality of the coefficients $\Lambda^{(\mathcal{G} ; a, b)}$ in equation (1.1). We are also able to observe that the physics of these models is expressible in terms of the physics of the corresponding non-affine models albeit in a non-trivial way. Indeed the quantities $\check{\omega}^{-r} \bar{\phi}_{b}$ and $\Delta_{b}^{(r)}$ are determined wholly within the structure of the Lie algebra $\bar{g}$ without reference to the peculiarities of the greater KacMoody algebra $\widehat{\mathrm{g}}$. These quantities provide one with an exceptionally quick (i.e. finite) method of evaluating the partition function of any affine model. What initially appears to be an infinite sequence, may in fact be reduced to a finite number of evaluations by equation (6.25). It might be possible that this can be used as a mechanism to collect the degenerate $c=1$ Virasoro characters in the partition functions in such a way as to observe the action of higher symmetries, such as supersymmetry or those associated with $W$-algebras, in the physics of these models. We remark that neither of these results are apparent from the purely algebraic expression (4.12).

Most importantly, the geometric result (5.17) and its consequences lend support to the idea that the geometry of root systems has a more general role within conformal field theories and related structures. This idea has been investigated for $S$-matrices by Dorey in [4] and [5]; and for more general graph-lattice models by Zuber [27].

\section{Acknowledgements}

This work is a subset of work carried out by the author between April 1995 and October 1998 in partial fulfillment of the requirements for the award of the degree of Doctor of Philosophy of the University of Durham (England). A full account of this and other work may be found elsewhere [26].

The author wishes to express his thanks to Patrick Dorey for supervising this project and for numerous valuable discussions. 


\section{References}

[1] S. Berman, Y. S. Lee, R. V. Moody, J. Algebra 121 (1989), 339-357.

[2] J. Cardy, Nucl. Phys. B240 (1984), 514-532; Nucl. Phys. B270 (1986), 186-204; Nucl. Phys. B275 (1986), 200-218; Nucl. Phys. B324 (1989), 581-596.

[3] A. Coleman, Invent. Math. 95 (1989), 447-477.

[4] P. Dorey, Nucl. Phys. B358 (1991), 654-676; Nucl. Phys. B374 (1992), 741-761.

[5] P. Dorey, "Hidden geometrical structures in integrable models", a talk given at the conference "Integrable quantum field theories" held in Como, Italy, 13-19 September 1992; hep-th/9212143.

[6] P. Dorey, Int. J. Mod. Phys. A8 (1993), 193-208.

[7] P. Di Francesco, H. Saleur, J.-B. Zuber, Nucl. Phys. B285 (1987), 454-480.

[8] P. Di Francesco and J.-B. Zuber, Nucl. Phys. B338 (1990), 602-646.

[9] P. Di Francesco, Int. J. Mod. Phys. A7 (1992), 407-500.

[10] J. Fuchs, "Affine Lie algebras and quantum groups", Cambridge University Press, Cambridge, England (1995).

[11] P. Ginsparg, Nucl. Phys. B295 (1988), 153-170.

[12] P. Ginsparg, "Applied conformal field theory", in Les Houches, session XLIX, 1988, "Champs, cordes et phénomènes critiques", ed. by E. Brèzin and J. Zinn-Justin. Elsevier Science Publishers B.V., North-Holland, Amsterdam, The Netherlands (1989).

[13] J. Humphreys, "Introduction to Lie algebra and representation theory", SpringerVerlag, N.Y.C, New York (1972).

[14] J. Humphreys, "Reflection groups and Coxeter groups", Cambridge University Press, Cambridge, England (1990).

[15] V. Kac, "Contravariant form for infinite-dimensional Lie algebras and superalgebras", in "Lecture notes in physics 94", 441-445, Springer, N.Y.C., New York (1979).

[16] B. Kostant, Am. J. Math. 81 (1959), 973-1032.

[17] B. Kostant, Proc. Natl. Acad. Sci. USA 81 (1984), 5275-5277; Société Mathématique de France Astérisque, hors série, (1985), 209-255.

[18] B. Nienhuis, J. Stat. Phys. 34 (1984), 636-761.

[19] V. Pasquier, Nucl. Phys. B285 (1987), 162-172.

[20] V. Pasquier, J. Phys. A: Math. Gen. 20 (1987), L1229-L1237.

[21] H. Saleur, J. Phys. A: Math. Gen. 20 (1987), L1127-L1133; J. Phys. A: Math. Gen. 22 (1988), L41-48. 
[22] H. Saleur and M. Bauer, Nucl. Phys. B320 (1989), 591-624.

[23] N. Sochen, Nucl. Phys. B360 (1991), 613-640.

[24] R. Steinberg, Trans. Amer. Math. Soc. 91 (1959), 493-504.

[25] R. Steinberg, Pac. J. Math. 118 (1985), 587-598.

[26] R. Talbot, "Lattice models, cylinder partition functions, and the affine Coxeter element", University of Durham (England) Ph.D. thesis (1998).

[27] J.-B. Zuber, Commun. Math. Phys. 179 (1996), 265-294. 